\title{
Search Engine Optimization - Study and Analysis
}

\section{Srinithi $S^{a}$,}

${ }^{a}$ Programmer Analyst Trainee, Cognizant Technology Solutions, Coimbatore, Tamil Nadu, India.

* Corresponding Author: sripsgtech111@gmail.com

Received : $03^{\text {rd }}$ June 2020, Accepted : $24^{\text {th }}$ September 2020

Abstract: Search Engine Optimization is about understanding what people are searching for online, the answers they are seeking, the words they are using, and the type of content they wish to consume. Knowing the answers to these questions will allow you to connect to the people who are searching online for the solutions you offer. Building the onsite and offsite page tactics will help us to build a better site that ranks higher in search and also with better click through rates. Understanding what the users or consumers need and producing the same to them is one of the major factor in the Digital Marketing. Using Analytics tool we can analyze how our site is doing well or not with our intended action will help us to understand our audience better.

Keywords: SEO, Keyword, Links, Meta description, Title, Robot, Error, Query, Search, Online

\section{Introduction}

Search Engine Optimization is the practice of increasing both the quality and quantity of website traffic, as well as exposure to your brand, through non-paid search engine results.

In this paper I would like to discuss some of the techniques and tactics to how to enable high click through rates and also how to understand the user and make the site rank better with the better use of keywords.

\section{On Page SEO}

Search Engine Optimization is the practice of increasing both the quality and quantity of website traffic, as well as exposure to your brand, through non-paid search engine results.

\subsection{Title Tags}

The title tags are important for a page. I will explain the importance and the proper use of a Title tag with an example of a Title tag.

<title>UC Davis Continuing and Professional Education</title>

I have modified the above title Tag as below 
<title>Continuing and Professional Education - Online | UC Davis Extension</title>

1. The one after the $\mid$ symbol is the brand name and the usage of brand name is important and is useful for higher site ranking.

2. The title tag has to be 55 to 60 characters in length.

3. The title tag will have the keywords that user searches I used the term online so that when the user searches for online courses our page would definitely make a way through it and included the hyphens as it was long for the user to see.

4. If your title tag is long then use hyphens for optimization and for effective user search [1].

\subsection{Meta Description}

Meta descriptions provide the concise summaries of web pages. Let us look at an example and understand it better.

<meta name="description" content="GOOGLE SEO - BEST PRACTICES: Meta descriptions are HTML attributes that provide concise summaries of web pages. Meta description tags, while not tied to search engine rankings can have a positive effect on clickthrough rates. As for length, Google generally truncates meta descriptions snippets at...">

I have modified the above Meta description as below.

$<$ meta name="description" content="Meta descriptions provide the concise summaries of web pages. Meta descriptions also are important for high click through rates. Please have a look at Google SEO 2020 Meta Descriptions ">

1. Included better keywords for Meta description that makes the search better.

2. Also have included Call of Action which is nothing but telling the user what has to be done. It is believed that call of action generally makes the user to do the task that the site tells.

3. Have included updated year so that it increases the high click through rates. Often people look for the latest information.

4. Also the Meta description should not be too long with does not attract the user to the site [1].

\subsection{Heading Tags}

In order to understand about Heading Tags. I took the below website and analyzed the website for $\mathrm{H} 1$ and $\mathrm{H} 2$ tags.

\section{"https://www.webmd.com/diet/ss/slideshow-health-benefits-ginger"}

Found the below $\mathrm{H} 1$ and $\mathrm{H} 2$ tags

H1 TAGS: <h1>Health Benefits of Ginger $</$ hl > 
H2 TAGS: $<$ h2 $>$ Fights Germs $<$ h2 $><$ h2 $>$ Keeps Your Mouth Healthy $</$ h2 $><$ h2 $>$ Calms Nausea $</$ h2 $><$ h2 $>$ Soothes Sore Muscles $</$ h2 $><$ h2 $>$ Curbs Cancer Growth $<$ h2 $><$ h2 $>$ Lowers

Blood Sugar $</$ h2 $><$ h2 $>$ Eases Period Pains $<$ h2 $><$ h2 $>$ Protects Against Disease $</$ h2 $>$ $<\mathrm{h} 2>$ Relieves Indigestion $</ \mathrm{h} 2>$

There were more of $\mathrm{H} 2$ tags and it is better to keep it less or the user might get frustrated using the site. Understanding the user needs is one of the prime factor when considering the SEO.

\subsection{Using On Page SEO Tactics}

1. Making the content as unique as possible without any duplicate stuff.

2. To keep an eye on keywords that is once used too much can affect the click through rates. Hence optimize the use of keywords.

3. For the site to rank best it is advised to keep everything crisp and short not too long or too less. Meta descriptions and Title tags and URL are meant to be within a certain length and once exceeded can make the site rank less and can affect the user experience.

4. It is always better to use the original and unique content as much as possible. Understanding what the user needs is one of the major factor in SEO [2].

\section{Off Page/Off Site SEO}

The Offsite SEO strategy is of major importance for branding and social recognition. For the website design will incorporate the internal links as possible as so when the user searches the query the YouTube video related to it pops out on the ranking. Also for Instagram based on the feed likes and shares will make the content unique and admirable as possible to make the views high so as to make high branding.

\subsection{Link Building}

Some of the link building methods I would like to recommend for a website focused on pet care products.

1. Add an inbound link or a backlinks of a Pet Care Product store that is authoritative and is of high brand value so that customers can rely upon and shop.

2. Add an anchor text link inside the body of the content that links to the best pet care products online and that are most searched and bought in the recent years.

3. Avoid excessive link usage that might affect the user experience.

4. Make the content unique and link it to a better suitable content from another website that triggers the search ranking of the page [3].

\subsection{Off Page Tactics}

1. Adding backlinks to our site from the most relevant sites with good content. 
2. Gaining higher brand recognition and value through social media by making the post with unique content and by gaining audience.

3. Avoid too much of links so that might sound irritating to the user and must use the anchor text to link it to a site that is authoritative.

\section{Xml Sitemaps}

The XML Sitemap is for the search engines to recognize about the content of the page. It gives some useful information about the page so that the search engines can understand.

I created the XML site map using the Screaming Frog tool. I downloaded the free version and was able to crawl only upto 500 pages. If we needed to crawl more than 500 pages we should buy the paid version.

The sample of the XML Sitemap created:

<url>

$\langle$ loc $>$ https://learndigital.withgoogle.com/digitalgarage $</ l o c>$

$<$ lastmod $>2020-05-02</$ lastmod $>$

$<$ changefreq $>$ daily $</$ changefreq $>$

$<$ priority $>1.0<$ priority $>$

$</$ url $>$

Here the information such as when was the site last modified and the frequency at which it changes and the priority is mentioned in the XML Sitemap [4].

\section{Robot.Txt File}

I am using the below link for analyzing the robot.txt file and XML Sitemap "University of California Davis Health System"

User-agent: ${ }^{*}$

Disallow:

This file allows complete access of site by the robots. The Disallow field is left blank which provides the information to the robots that they can access all the directories below mentioned.

The * symbol denotes that the robot has to follow all the rules that is set below. The robot.txt file is always found in the root directory [5].

\subsection{Robot.Txt File - Complete}

Found the robot.txt file in the below path "https://health.ucdavis.edu/robots.txt":

\# robots.txt file for health.ucdavis.edu 
\# Thurs, 13 Oct 2016

\# Exclude Files From All Robots:

User-agent: *

Disallow:

/common/aboutus/

/common/amenities/

/common/appointments/

/common/awards/

/common/clinicaltrials/

/common/contactus/

/common/exec_leadership/

/common/facts_figures/

/common/features/

/common/health_tips/

/common/inthenews/

/common/intranet/

/common/lodging/

/common/map/

/common/medcenter_som_leadership/

/common/nursing_leadership/

/common/pavilion/

/common/profiles/

/common/promocarousel/

/common/publications/publications.html

/common/robotic_surgery/

/common/sacarea/

\# End robots.txt file 


\section{Technical SEO}

The things I would like to incorporate into the website regarding Technical SEO are

1. To crawl the site using the screaming frog tool or any other tool and finding the sites that are of error status and analyzing the sites so that they rank higher than the other sites.

2. Analyzing the XML Sitemaps and able to prioritize the important URL and make it modified for better user experience. Since XML Sitemaps provide the information to the search engines.

3. Using robot.txt file and by giving the better information to the robots of what or what not to access.

4. Taking into account the HTTP error as important as possible since they have a greater impact on the user likeliness of the site. By using the SEO tools be able to crawl the site and identify the error and make the errors as less as possible so that makes the site rank higher.

5. By using Google search console the webmaster can analyse the web page and can correct the error codes.

6. Also use the redirects once when the page is not found. Also use the order of redirect. When the page is not available then redirect it to a similar specific page.

1. By using the above tactics it would definitely make the website rank higher and make the search engines identify the page [6].

\subsection{Pesky Error Codes}

200 - Successful which means the page is loaded properly

404 - Page not found error code (Page was removed or no longer exists)

Soft 404 - Page is no longer available but still it shows that the page is successful (200).This happens when the content of the page is removed but not the page.

500 - Server error. This is a temporary issue and it will correct itself. If not the website developer must see through the server logs and find the issue and fix it.

503 - Service is unavailable. This error code will be produced when the server is down or during maintenance or when the server is overloaded.

\subsection{Redirects 101}

Whenever we are needed to remove the page or update the page to a newer version it is better to use a redirect that tells the search engine how to carry forward the site.

\subsubsection{Types of Redirect:}

Permanent Redirect/HTTP 301 - Tells the search engine that that the page no longer exist and the redirected site is a permanent one and will transfer all the credits to the new page. 
Temporary Redirect/HTTP 302 - Here it will tell the search engines that the redirect is temporary and limited one and will not transfer the credits.

Metarefresh - No HTTP code is presented with metarefresh. Redirect is executed by the page level rather than the server.

Generally 301 redirect is preferred most than the 302 redirect. Chaining redirects is not recommended since some authority is

lost with each redirect. It is preferable to redirect to a specific page rather than a category page or site home [6].

\section{Search Query}

1. Navigational Query- User knows what he is searching mostly a website he has already enjoyed watching the content. For example: The search query is amazon

2. Informational Query - People look for a bit of information.

3. Transactional query - When the user is ready to buy and they look for search results from google to what they search for.

\section{Buyers Persona}

I would like to take the Wedding Planner site for creating Ideal Buyers Persona. Please find the link to the Site below "https://www.wedmegood.com/".Must take the below things into consideration when analyzing a person as shown in Figure 1.

Here I would take two personas into account - A Male Persona and a Female Persona.

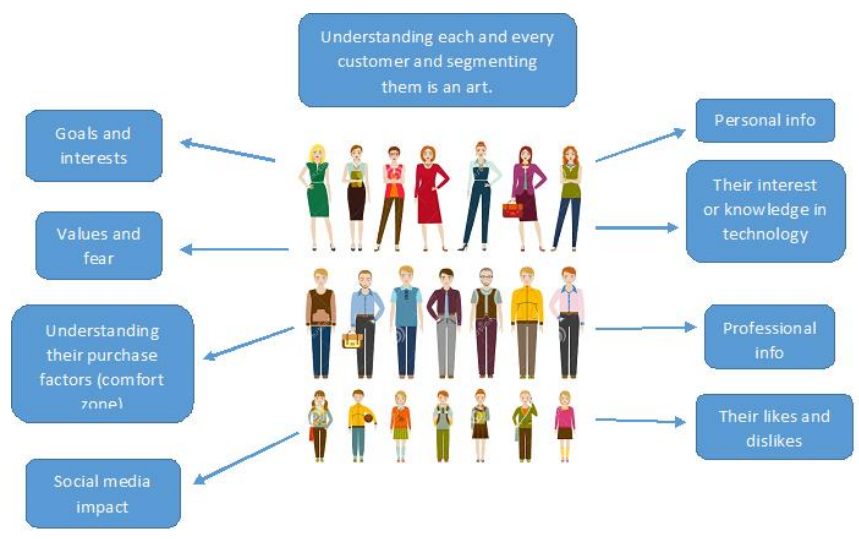

Figure 1. Buyers Persona 


\subsection{Male Persona}

\section{Male Persona: - Simple, Cheap and best, Techie, Cost Effective, Traditional}

$>$ The male Persona is identified to be simple and we would suggest him some dresses that would be of simple clothing line yet a grand one that makes him satisfied. Mostly as he eyes on simple one will include more collections of cotton brands.

$>$ Since the male Persona focuses on cost effective yet best wedding will show him the better Pocket Friendly stunner packages that best suit him.

$>$ Since the male persona holds a high traditional value will make the decorations such that it includes wedding pandits and the decorations takes a high pitch with the traditions.

As a techie the male persona will be towards photography and stuffs. Hence a cost effective drone to capture the overall wedding will make him like the page more

Analyzing the male persona the better keywords that would make the site rank higher would be:

Pocket Friendly, Simple costume, Cotton, Traditional decorations.

\subsection{Female Persona}

\section{Female Persona: - Fairy tale Wedding, Grand, Lively, Dramatic}

$>$ The Female persona dreams of a Fairy tale wedding so the best suggestion to opt for would be a destination wedding that would be the best option to attract the audience.

$>$ Since from her persona she believes in the grandeur of the wedding hence a perfect mehendi ceremony with music and dance would be her type and our suggestions should include the best mehendi artist and the best $\mathrm{DJ}$ in town to make the user stay interested in the page.

$>$ This persona believes that the wedding is a onetime opportunity to be visualized by many and hence a photo-shoot at the destination wedding would be a better suggestion.

$>$ Coming on to the Dresses the bridal will look upto a grand and beautiful dress that carries its own charisma and would suggest her the bridal lehengas and Gowns of her choice.

Analyzing the Female persona the better keywords that would make the site rank higher would be:

Destination Wedding, Bridal Lehengas, Sangeet, Mehendi Ceremony, Photoshoot [7]. 


\section{Results and Discussion}

Analyzed the GOOGLE domain using the Mozbar tool and came up with the below conclusions.

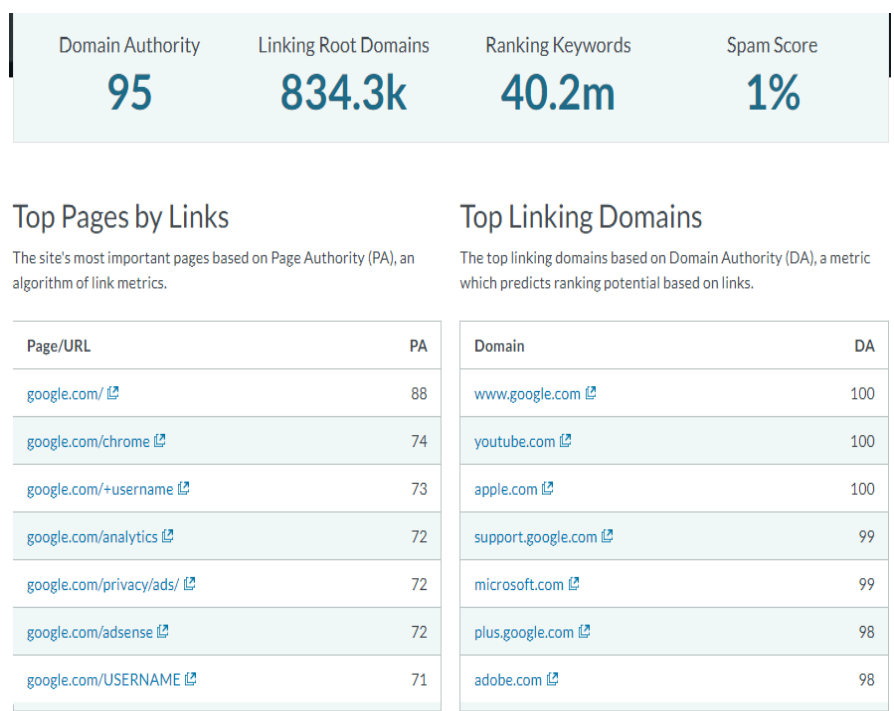

\section{Figure 2. Google Domain Authority}

Google has the highest domain authority and branding and there are a list of top pages based on Page authority as shown in Figure 2

\begin{tabular}{|c|c|c|c|}
\hline \multicolumn{2}{|c|}{$\begin{array}{l}\text { Keywords by Estimated Clicks } \\
\text { Estimated clicks for top keywords, based on volume and CTR. }\end{array}$} & \multicolumn{2}{|l|}{$\begin{array}{l}\text { Top Ranking Keywords } \\
\text { Your top keywords sorted by ranking position. }\end{array}$} \\
\hline Keyword & Visibility & Keyword & Rank \\
\hline g'mai'l & $37,693,970$ & google & 1 \\
\hline google classroom & $36,490,971$ & gmail & 1 \\
\hline gmail & $35,688,971$ & g'mai"l & 1 \\
\hline 'google docs' & $34,886,972$ & google maps & 1 \\
\hline google docs & $34,886,972$ & google maps & 1 \\
\hline googledrive & $34,886,972$ & google translate & 1 \\
\hline 'google drive' & 33,683,973 & translate & 1 \\
\hline
\end{tabular}

Figure 3. Keywords Classification

As we all know keywords are really important for SEO site ranking. Proper selection and usage of keywords is essential for proper site ranking. Figure 3 emphasizes the top keywords that is sorted in the google domain. 


\begin{tabular}{|lcr|}
\hline Domain & Domain Authority & Visibility \\
\hline www.google.com & 100 & 19.59 \\
\hline play.google.com & 99 & 7.29 \\
\hline translate.google.com & 94 & 4.79 \\
\hline support.google.com & 99 & 4.42 \\
\hline store.google.com & 90 & 4.16 \\
\hline maps.google.com & 98 & 3.64 \\
\hline apps.apple.com & 100 & 2.21 \\
\hline
\end{tabular}

Figure 4. Competitor Analysis

Analyzing our competitor is a part of SEO as shown in Figure 4. Keeping a Unique selling point (USP) profile and understanding our competitors is a part of our business.

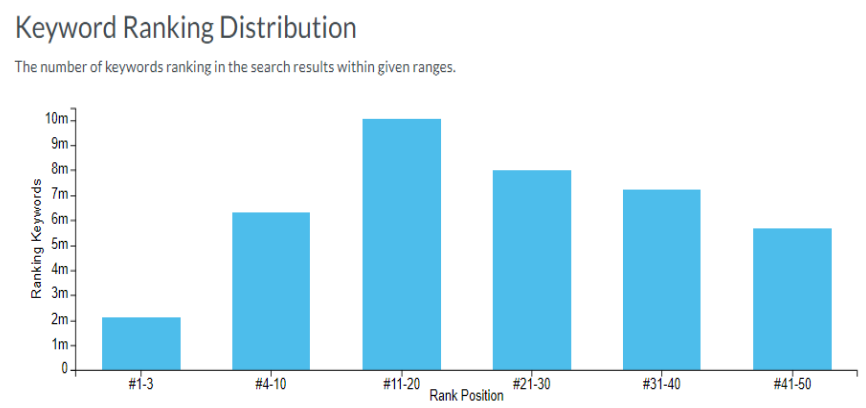

Figure 5. Keyword Distribution

The proper and correct usage of keywords not too many or less is important for site ranking. Figure 5 shows the keyword ranking distribution for Google site.

\section{Conclusion}

Set goals of what you need from being online and work towards it. Use analytics tool to track what is right and how we are moving forward in the digital presence. Plan accordingly. Knowing what we need and planning and working accordingly help us to achieve greater in this field. Usage of better keywords and knowing well with the search will help stay in business and will make the site rank higher.

\section{References}

[1] Zhen Liu and Philippe Nain, "Optimization issues in Web search Engines", IBM research,. 2006, VI, 981- 1015, DOI: 10.1007/978-0- 387-30165-5_34 Zhen Liu and Philippe Nain, “Optimization issues in Web search Engines”, IBM research, 2006, VI, 981- 1015, DOI: 10.1007/978-0-387-30165-5_34. 
[2] Fuxue Wang; Yi Li; Yiwen Zhang; Coll. of Econ. \& manage, "An empirical study on the search engine optimization technique and its outcomes," Artificial Intelligence, Management Science and Electronic Commerce (AIMSEC), Aug. 2011.

[3] (2011)"Search Engine Ranking Factors V2". SEOmoz.org [Online]. http://www.seomoz.org/article/searchranking-factors.

[4] S.Mukherjee, "A probabilistic model for optimal searching of the deep Web”, 2003.

[5] Danny Sullivan (June 14, 2004). "Who Invented the Term "Search Engine Optimization"?". Search Engine Watch. Retrieved May 14, 2007. See Google groups thread.

[6] "Search King, Inc. v. Google Technology, Inc., CIV-02- 1457-M" (PDF). docstoc. com. May 27, 2003. Retrieved May 23, 2008.

[7] "Technology \& Marketing Law Blog: KinderStart v. Google Dismissed-With Sanctions Against KinderStart's Counsel". blog.ericgoldman.org. Retrieved June 23, 2008.

\section{Acknowledgements: NIL}

Conflict of interest: NIL

About the License: (c) 2020 The Author. This work is licensed under a Creative Commons Attribution 4.0 International License which permits unrestricted use, provided the original author and source are credited. 